\title{
ON SOME CONTACT PROBLEMS FOR BODIES WITH ELASTIC INCLUSIONS
}

\author{
N. SHAVLAKADZE
}

\begin{abstract}
The paper deals with the contact problems of the theory of elasticity. The problems are reduced to Prandtl-type integral differential equations with a coefficient at the singular operator which has higher-order zeros at the ends of the integration interval. In some concrete cases the solution is constructed efficiently. Asymptotic representations are obtained.
\end{abstract}

Investigation of the problem of stress concentration when contact between different elastic media takes place remains one of the most topical tasks. Inclusions such as punches and cuts are stress concentrators and hence the study of the influence of inclusions on the stress-strained state of solid deformable bodies, as well as the elaboration of methods allowing one to reduce stress concentration, are of great theoretical and practical importance. The problems of contact interaction between rigid inclusions of various geometrical forms and elastic bodies are considered in [1-5]. In [6] some problems of contact interaction between a piecewise-homogeneous plane and a rigid inclusion, and also the anti-plane problem of an elastic half-space and problems of bending of plates with thin-shelled inclusions, are reduced to integral equations by means of the generalized method of integral transforms.

As distinct from the previous papers, this paper deals with the problems of contact interaction between a thin elastic inclusion of varying rigidity and (a) an elastic half-space which is in the state of anti-plane deformation, and (b) an elastic piecewise-homogeneous plane.

1991 Mathematics Subject Classification. 45J05, 73C02.

Key words and phrases. Interface problem, elastic inclusion, Prandtl type equation. 285

1072-947X/98/0500-0285\$15.00/0 @ 1998 Plenum Publishing Corporation 


\section{$\S 1$. Anti-Plane Problem for an Elastic Half-Plane with an ELASTIC INCLUSION}

Let an elastic strip-like inclusion $0 \leq y \leq b,-\infty<z<\infty$ lying in the plane $x=0$ be inserted into the elastic half-space $(-\infty<x, z<\infty, y \geq 0)$ with the boundary free from stresses. It is required to find a field of stresses and displacements if a shearing load of intensity $\tau_{0}$ acting along the $O z$ axis is applied to the outer edge of the above-mentioned inclusion. The displacement $\omega$ along the $O z$-axis is assumed to be different from zero, and $\omega=\omega(x, y)$. The tangential stresses $\tau_{y z}=\gamma \frac{\partial \omega}{\partial y}$ and $\tau_{x z}=\gamma \frac{\partial \omega}{\partial x}$, where $\gamma$ is the shear modulus, are also assumed to be different from zero.

The problem formulated above is equivalent to the boundary value problem for the Laplace equation

$$
\frac{\partial^{2} \omega}{\partial x^{2}}+\frac{\partial^{2} \omega}{\partial y^{2}}=0, \quad \frac{\partial \omega(x, 0)}{\partial y}=0, \quad(x, y) \in D,
$$

where $D:\{(x, y)|| x \mid<\infty, y \geq 0\} \backslash\{0, y) \mid 0 \leq y \leq b\}$; it is required to find, for problem (1.1), a solution vanishing at infinity, i.e.,

$$
\lim _{|x| \rightarrow \infty} \omega(x, y)=0 \text { and } \lim _{y \rightarrow+\infty} \omega(x, y)=0 .
$$

In passing through the inclusion, the tangential stress $\tau_{x z}$ undergoes discontinuity, while the displacements are continuous:

$$
\begin{aligned}
<\tau_{x z}(0, y)>= & \gamma<\frac{\partial \omega(0, y)}{\partial x}>=\mu(y), \quad \mu(y) \equiv 0, \quad y>b, \\
& <\omega(0, y)>=0, \quad 0 \leq y \leq b,
\end{aligned}
$$

where $<f(0, y)>=f(-0, y)-f(-0, y)$.

The equation of equilibrium on the $(0, y)$-part of the inclusion is of the form

$$
\frac{d \omega(y)}{d y}=-\frac{1}{E(y)}\left[\tau_{0}-\int_{0}^{y} \mu(t) d t\right], \quad 0<y<b
$$

while the condition of equilibrium of the inclusion has the form $\int_{0}^{b} \mu(t) d t-$ $\tau_{0}=0$, where $E(y)=\frac{E_{0}(y) h_{0}(y)}{1-\nu_{0}^{2}}, E(y) \geq 0$. Here $E_{0}(y)$ and $\nu_{0}$ are, respectively, the modulus of elasticity and the Poisson coefficient for the material with an inclusion of small thickness $h_{0}(y)$.

Theorem 1.1. If problem (1.1), (1.2), (1.3) has a solution, then it is unique. 
Proof. Suppose that the problem has two solutions. Then the "difference" of these solutions satisfies the basic equations in the absence of external forces, i.e., for $\tau_{0} \equiv 0$.

Consider the integral

$$
\mathcal{J}=\iint_{S_{h}}\left(X_{n} u+Y_{n} v+Z_{n} w\right) d s
$$

where $S_{h}$ is the boundary of the domain obtained by intersection of the half-space with the planes $L_{h}(z=h)$ and $L_{-h}(z=-h) ; X_{n}, Y_{n}, Z_{n}, u, v$, $w$ are the components of external stresses and displacements on the surface $S_{h}$.

By the Ostrogradskii-Green formula we obtain

$$
\begin{gathered}
\iint_{S_{h}}\left(X_{n} u+Y_{n} v+Z_{n} w\right) d s=\iiint_{V_{h}}\left\{\lambda \theta^{2}+2 \mu\left(e_{x x}^{2}+e_{y y}^{2}+e_{z z}^{2}+\right.\right. \\
\left.\left.+2 e_{x y}^{2}+2 e_{x z}^{2}+2 e_{y z}^{2}\right)\right\} d x d y d z
\end{gathered}
$$

where $\theta=e_{x x}+e_{y y}+e_{z z}, e_{x x}, e_{y y}, e_{z z}, e_{x y}, e_{y z} e_{x z}$, are the deformation components and $\lambda, \mu$ are the Lamé constants for the half-space material.

For problem (1.1), (1.2) the integral on the left-hand side of (1.4) takes the form

$$
\begin{aligned}
\iint_{S_{h}}\left(X_{n} u+Y_{n} v\right. & \left.+Z_{n} w\right) d s=\int_{-h}^{h} d z \int_{0}^{b}<\tau_{x z}(0, y)>\omega(y) d y+ \\
& +\iint_{L_{h}} Z_{n} \omega d s+\iint_{L_{-h}} Z_{n} \omega d s
\end{aligned}
$$

Since the outer normals of the planes $L_{h}$ and $L_{-h}$ are directed counterwise, we have $\iint_{L_{h}} Z_{n} \omega d s=-\iint_{L_{-h}} Z_{n} \omega d s$. Using condition (1.3), we finally get

$$
\begin{gathered}
\int_{-h}^{h} d z \int_{0}^{b}<\tau_{x z}(0, y)>\omega(y) d y=\int_{-h}^{h} d z \int_{0}^{b} \mu(y) \omega(y) d y= \\
=2 h \int_{0}^{b} \omega(y) d\left(\int_{0}^{y} \mu(t) d t\right)=\left.2 h \omega(y) \int_{0}^{y} \mu(t) d t\right|_{0} ^{b}- \\
-2 h \int_{0}^{b} \omega^{\prime 2}(y) E(y) d y=-2 h \int_{0}^{b} E(y) \omega^{\prime 2}(y) d y
\end{gathered}
$$

Since the integrand on the right-hand side of (1.4) is a positive definite quadratic form, in view of representation $\left(1.4_{0}\right)$, we can conclude that $\mathcal{J}=$ 0 . This means that in the absence of external forces $\mu(y)=0$, i.e., the homogeneous problem admits only the trivial solution. 
Let us represent the harmonic function $\omega(x, y)$ in the form

$$
\omega(x, y)=\varphi(z)+\overline{\varphi(z)}, \quad z=y+i x,
$$

where $\varphi(z)$ is an analytic function in the half-plane $\operatorname{Re} z>0$ cut along the segment $[0, b]$.

Conditions (1.2) yield

$$
\begin{aligned}
& {\left[\varphi^{\prime}(y)+\overline{\varphi^{\prime}(y)}\right]^{+}-\left[\varphi^{\prime}(y)+\overline{\varphi^{\prime}(y)}\right]^{-}=0,} \\
& {\left[\varphi^{\prime}(y)-\overline{\varphi^{\prime}(y)}\right]^{+}-\left[\varphi^{\prime}(y)-\overline{\varphi^{\prime}(y)}\right]^{-}=-\frac{\mu(y)}{i \gamma} .}
\end{aligned}
$$

By adding these equations we obtain the boundary value problem of linear conjugation:

$$
\varphi^{\prime+}(y)-\varphi^{\prime-}(y)=-\frac{\mu(y)}{2 i \gamma}, \quad 0<y<b .
$$

By condition (1.1), $\varphi^{\prime}(i x)+\overline{\varphi^{\prime}(i x)}=0$. After considering the function

$$
\Psi(z)= \begin{cases}\varphi^{\prime}(z) & \text { for } \quad \operatorname{Re} z>0 \\ -\overline{\varphi^{\prime}(\bar{z})} & \text { for } \quad \operatorname{Re} z<0\end{cases}
$$

condition (1.5) takes the form

$$
\Psi^{+}(y)-\Psi^{-}(y)=-\frac{\mu_{1}(y)}{2 i \gamma}, \quad|y|<b,
$$

where $\mu_{1}(y)=\left\{\begin{array}{l}\mu(y), 0 \leq y<b \\ \mu(-y),-b<y<0\end{array}\right.$.

Since $\Psi(z)$ is analytic on the whole plane cut along the segment $(-b, b)$, the solution of the boundary value problem (1.7) vanishing at infinity can be given by the formula [7]

$$
\Psi(z)=-\frac{1}{4 \pi \gamma} \int_{-b}^{b} \frac{\mu_{1}(t) d t}{t-z} .
$$

Taking into consideration (1.6) and (1.7), the condition for inclusion (1.3) takes the following form:

$$
\frac{1}{2 \pi \gamma} \int_{-b}^{b} \frac{\mu_{1}(t) d t}{t-y}=\frac{1}{E(y)}\left[\tau_{0}(y)-\int_{0}^{y} \mu_{1}(t) d t\right],|y|<b,
$$

where $\tau_{0}(y)=\left\{\begin{array}{l}\tau_{0}, 0 \leq y<b \\ -\tau_{0},-b<y<0,\end{array} \quad E(y)=E(-y)\right.$. Here we have continued in an odd manner equation (1.4) to the negative values of the argument. 
Introducing the notation $\int_{0}^{y} \mu_{1}(t) d t \equiv \nu(y)$ and taking into account the fact that the function $\mu_{1}(y)$ is even, we obtain the Prandtl-type integral differential equation

$$
\frac{\nu(y)}{E(y)}+\frac{1}{2 \pi \gamma} \int_{-b}^{b} \frac{\nu^{\prime}(t) d t}{t-y}=\frac{\tau_{0}(y)}{E(y)}, \quad-b<y<b,
$$

provided that $\nu(-b)=-\tau_{0}, \nu(b)=\tau_{0}$.

\section{$\S 2$. Composite Plane Reinforced with an Inclusion of Finite \\ LENGTH}

We consider a composite elastic plane by which is meant an unbounded elastic medium composed of two half-planes $(y>0$ and $y<0)$ and having different elastic constants $\left(E_{+}, \mu_{+}\right.$and $\left.E_{-}, \mu_{-}\right)$. They are assumed to be in a state of plane deformation and are reinforced on the segment $(-b, b)$ with an inclusion of varying thickness $h_{0}(x)$, with the modulus of elasticity $E_{0}(x)$ and the Poisson coefficient $\nu_{0}$.

By the inclusion will be meant a thin plate to which vertical and horizontal forces of intensity $p_{0}(x)$ and $\tau_{0}(x)$, respectively, are applied. These functions are continuous on the segment $[-b, b]$; note that $P_{0}(x) \equiv 0$ and $\tau_{0}(x) \equiv 0$ for $x \neq(-b, b)$. When passing through the segment $(-b, b)$, the stress field undergoes discontinuity, while when passing through the remainding part of the $O x$-axis the stress and displacement fields remain continuous.

The basic equations of equilibrium of the $(-b, x)$-part of the inclusion are

$$
\begin{aligned}
& E_{1}(x) \frac{d u_{1}(x)}{d x}=\int_{-b}^{x}\left[\tau(t)-\tau_{0}(t)\right] d t, \quad-b<x<b, \\
& \frac{d^{2}}{d x^{2}} D_{1}(x) \frac{d^{2} v_{1}(x)}{d x^{2}}=p_{0}(x)-p(x),
\end{aligned}
$$

where

$$
\begin{array}{ll}
\tau(x)=\tau^{-}(x)-\tau^{+}(x), & E_{1}(x)=\frac{E_{0}(x) h_{0}(x)}{1-\nu_{0}^{2}}, \\
p(x)=p^{-}(x)-p^{+}(x), & D_{1}(x)=\frac{E_{0}(x) h_{0}^{3}(x)}{12\left(1-\nu_{0}^{2}\right)},
\end{array}
$$

$p^{ \pm}(x)$ and $\tau^{ \pm}(x)$ are respectively the unknown normal and tangential contact stresses on the upper and the lower contour of the inclusion, and $u_{1}$ and $v_{1}$ are the displacements of its points in the horizontal and vertical directions, respectively. $E_{1}(x)$ and $D_{1}(x)$ are positive functions from the general class of functions. 
The conditions of equilibrium of the inclusion are of the form

$$
\begin{gathered}
\int_{-b}^{b} \tau(t) d t=\int_{-b}^{b} \tau_{0}(t) d t \equiv T_{0}, \quad \int_{-b}^{b} p(t) d t=\int_{-b}^{b} p_{0}(t) d t \equiv P_{0}, \\
\int_{-b}^{b} t p(t) d t=\int_{-b}^{b} t p_{0}(t) d t \equiv M_{0} .
\end{gathered}
$$

Here the fact that the end sections of the inclusion are free from external loads is taken into account. We also take into consideration the condition of compatibility of deformations both of the inclusion and of the elastic composite plane with a defect along the segment $(-b, b)$.

Using the stress function $F(x, y)$ satisfying the biharmonic equation [7]

$$
\Delta^{2} F(x, y)=0,
$$

we obtain the formulas for stresses

$$
\begin{gathered}
\sigma_{x}=\frac{\partial^{2} F}{\partial y^{2}}, \quad \sigma_{y}=\frac{\partial^{2} F}{\partial x^{2}}, \quad \tau_{x y}=-\frac{\partial^{2} F}{\partial x \partial y}, \\
u^{\prime}=c \frac{\partial^{2} F}{\partial y^{2}}-d \frac{\partial^{2} F}{\partial x^{2}}, \quad v^{\prime \prime}=-c \frac{\partial^{3} F}{\partial y^{3}}-(2 c+d) \frac{\partial^{3} F}{\partial x^{2} \partial y},
\end{gathered}
$$

where

$$
c, d=\left\{\begin{array}{ll}
c_{+}, d_{+}, & y>0, \\
c_{-}, d_{-}, & y<0,
\end{array} c_{ \pm}=\frac{1-\mu_{ \pm}^{2}}{E_{ \pm}}, \quad d_{ \pm}=\frac{\mu_{ \pm}\left(1-\mu_{ \pm}\right)}{E_{ \pm}} .\right.
$$

Applying the Fourier transform to equations (2.3) and (2.4), we arrive at the homogeneous equation

$$
F_{j}^{(I V)}(y)-2 \alpha^{2} F_{\alpha}^{(I I)}(y)+\alpha^{4} F_{\alpha}(y)=0, \quad y \neq 0,
$$

with the boundary conditions

$$
\begin{gathered}
i \alpha<F_{\alpha}^{\prime}(0)>=\tau_{\alpha}, \quad<c F^{\prime \prime}{ }_{\alpha}+d \alpha^{2} F_{\alpha}>=0, \\
-\alpha^{2}<F_{\alpha}(0)>=p_{\alpha}, \quad<c F^{\prime \prime \prime}{ }_{\alpha}+(2 c+d) \alpha^{2} F_{\alpha}^{\prime}>=0,
\end{gathered}
$$

where the transforms $\left\|F_{\alpha}(y), \tau_{\alpha}, p_{\alpha}\right\|=\int_{-\infty}^{\infty}\|F(x, y), \tau(x), p(x)\| e^{i \alpha x} d x$.

Using the arguments from [6], we construct a solution of the one-dimensional discontinuous boundary value problem $(2.5),(2.6)$ in the form

$$
F_{\alpha}(y)=\sum_{j=0}^{3} x_{j} \widetilde{Y}_{j}(y)
$$


where $\widetilde{y}_{j}(y)$ is the base function of discontinuous solutions,

$$
\begin{aligned}
& \widetilde{y}_{j}(y)=\alpha^{4} E_{\alpha}^{(-1-j)}(y), \quad j=0,1 ; \quad \widetilde{y}_{j}(y)=-E_{\alpha}^{(3-j)}(y), \quad j=2,3 . \\
& E_{\alpha}^{(0)}(y)=e_{\alpha}(y), \\
& E_{\alpha}^{(n)}(y)=\frac{1}{4}(-\operatorname{sgn} y)^{n}[|\alpha y|-(n-1)] \alpha^{n-3} e^{-|\alpha y|}, \quad n= \pm 1, \pm 2, \ldots .
\end{aligned}
$$

The function $e_{\alpha}(y-\xi)$ defined by the formula $e_{\alpha}(y)=\frac{1+|\alpha y|}{4|\alpha|^{2}} e^{-|\alpha y|}$ is taken as the Green's function.

Having fulfilled the conjugate conditions (2.6), we get

$$
\begin{aligned}
& x_{1}=-\alpha^{-2} p_{\alpha}, \\
& x_{1}=-i \alpha^{-1} \tau_{\alpha},
\end{aligned} \quad a_{k 2} x_{2}+a_{k 3} x_{3}=-a_{k 0} x_{0}-a_{k 1} x_{1},
$$

where $a_{k j}=<\widetilde{y}_{j}^{(k)}(0)>\quad k=0,1$.

$$
a_{k j}=<\widetilde{y}_{j}^{(k)}(0) c-\alpha^{2} \widetilde{y}_{j}^{(k-2)}(0)\left[c-(-1)^{k}(c+d)\right]>, \quad k=2,3 .
$$

For our formulas to be more compact, we introduce the functionals $V_{k}^{(j)}\left(f_{n}\right), k=0,1$, acting on the sequence $f_{n}$ :

$$
\begin{aligned}
c_{0} V_{0}^{(j)}\left[f_{n}\right] & =c_{2} f_{j}-\left(c_{1}-c_{0}\right) f_{j+1}-c_{0} f_{j-1}, \\
c_{0} V_{1}^{(j)}\left[f_{n}\right] & =\left(c_{1}+c_{0}\right) f_{j}-c_{2} f_{j+1}-c_{0} f_{j-2},
\end{aligned}
$$

where the numbers $c_{0}, c_{1}, c_{2}$ represent the following combinations of the elastic constants:

$$
\begin{aligned}
c_{0} & =4\left(c_{+}+c_{-}\right)^{2}-\left[\left(c_{-}-c_{+}\right)-\left(d_{-}-d_{+}\right)\right]^{2} \\
c_{1} & =4\left(c_{+}+c_{-}+d_{+}+d_{-}\right)\left(c_{+}+c_{-}\right)+ \\
& +2\left[\left(c_{-}-c_{+}\right)-\left(d_{-}-d_{+}\right)\right]\left[c_{-}-c_{+}+d_{-}-d_{+}\right] \\
c_{2} & =2\left(c_{+}+c_{-}+d_{+}+d_{-}\right)\left[\left(c_{+}+c_{-}\right)-\left(d_{-}-d_{+}\right)\right]+ \\
& +4\left(c_{+}+c_{-}\right)\left(c_{-}-c_{+}+d_{-}-d_{+}\right) .
\end{aligned}
$$

Having defined $x_{j}(j=\overline{0,3})$ from (3.8) and substituted their values in (2.7), by means of the above introduced functionals (2.9) we can write

$$
F_{\alpha}(y)=V_{0}^{(0)}\left[|\alpha|^{1-n} E_{\alpha}^{(n)}(y)\right] p_{\alpha}+i \operatorname{sgn} \alpha V_{1}^{(0)}\left[|\alpha|^{1-n} E_{\alpha}^{(n)}(y)\right] \tau_{\alpha} .
$$

Substituting the values of transforms (2.10) in the inversion formulas and taking into account formulas (2.6) and also the convolution theorem, 
we obtain

$$
\begin{aligned}
\sigma_{x} & =\int_{-b}^{b} R_{1}^{(0)}(x-\xi, y) p(\xi) d \xi+\int_{-b}^{b} R_{2}^{(0)}(x-\xi, y) \tau(\xi) d \xi \\
\tau_{x y} & =\int_{-b}^{b} R_{1}^{(1)}(x-\xi, y) p(\xi) d \xi+\int_{-b}^{b} R_{2}^{(1)}(x-\xi, y) \tau(\xi) d \xi \\
u^{\prime} & =\int_{-b}^{b} R_{1}^{(2)}(x-\xi, y) p(\xi) d \xi+\int_{-b}^{b} R_{2}^{(2)}(x-\xi, y) \tau(\xi) d \xi \\
v^{\prime} & =\int_{-b}^{b} R_{1}^{(3)}(x-\xi, y) p(\xi) d \xi+\int_{-b}^{b} R_{2}^{(3)}(x-\xi, y) \tau(\xi) d \xi
\end{aligned}
$$

where

$$
\begin{aligned}
& R_{k}^{(m)}(x, y)=-\left(\alpha_{k}\right)^{m} V_{k}^{(m)}\left[K_{n}^{\left(s_{k-m}\right)}\right], \quad m=0,1 \\
& \left(\alpha_{k}\right)^{m} R_{k}^{(m)}(x, y)=c V_{k}^{(m)}\left[K_{n}^{\left(s_{k-m}\right)}\right]-\left[c+\alpha_{m}(c+d)\right] V_{k}^{(m-2)}\left[K_{n}^{\left(s_{k-m}\right)}\right], \\
& \quad m=2,3, \quad \alpha_{k}=(-1)^{k+1}, \quad s_{k}=\sin ^{2} \frac{\pi k}{2} \\
& K_{n}^{(0)}(x, y)=\frac{(-\operatorname{sgn} y)^{n}|y|}{4 \pi\left(x^{2}+y^{2}\right)}\left[\frac{y^{2}-x^{2}}{y^{2}+x^{2}}-(n-1)\right], \\
& K_{n}^{(1)}(x, y)=\frac{(-\operatorname{sgn} y)^{n} x}{4 \pi\left(x^{2}+y^{2}\right)}\left[\frac{2 y^{2}}{y^{2}+x^{2}}-(n-1)\right], \quad n=0, \pm 1, \pm 2, \ldots
\end{aligned}
$$

With the help of the above obtained discontinuous solution (2.11) we can get limiting values of the displacement field components as $y \rightarrow 0$. As a result, we obtain

$$
\begin{gathered}
u^{\prime}(x)=A p(x)+\frac{B}{\pi} \int_{-b}^{b} \frac{\tau(t) d t}{t-x}, \\
v^{\prime}(x)=-A \tau(x)+\frac{B}{\pi} \int_{-b}^{b} \frac{p(t) d t}{t-x}
\end{gathered}
$$

where

$$
\begin{aligned}
& A=\frac{a_{+} b_{-}\left(b_{+}+a_{-}\right)-a_{-} b_{+}\left(b_{+}+a_{-}\right)}{2 c_{0}}, \\
& B=\frac{a_{+} b_{-}\left(b_{+}+a_{-}\right)+a_{-} b_{+}\left(b_{+}+a_{-}\right)}{2 c_{0}} \\
& a_{ \pm}=3 c_{ \pm}-d_{ \pm}, \quad b_{ \pm}=c_{ \pm}+d_{ \pm} .
\end{aligned}
$$

Taking into account the contact conditions

$$
u^{\prime}(x)=u_{1}^{\prime}(x)
$$




$$
v^{\prime}(x)=v_{1}^{\prime}(x)
$$

and substituting formulas (2.12) in conditions (2.1), we arrive at the system of integral differential equations

$$
\begin{aligned}
& A \psi^{\prime \prime}(x)-\frac{B}{\pi} \int_{-b}^{b} \frac{\varphi^{\prime}(t) d t}{t-x}=\frac{\varphi(x)}{E_{1}(x)}+\frac{f_{1}(x)}{E_{1}(x)}, \quad-b<x<b, \\
& A \varphi^{\prime \prime}(x)+\frac{B}{\pi} \int_{-b}^{b} \frac{\psi^{\prime \prime \prime}(t) d t}{t-x}=\frac{\psi(x)}{D_{1}(x)}+\frac{f_{2}(x)}{D_{1}(x)},
\end{aligned}
$$

where

$$
\begin{aligned}
\varphi(x) & =\int_{-b}^{x} \tau(t) d t, \quad \psi(x)=\int_{-b}^{x} d t \int_{-b}^{t} p(\tau) d \tau \\
f_{1}(x) & =\int_{-b}^{k} \tau_{0}(t) d t, \quad f_{2}(x)=\int_{-b}^{x} d t \int_{-b}^{t} p_{0}(\tau) d \tau .
\end{aligned}
$$

Thus the solution of the above formulated problem is reduced to that of the system of integral differential equations (2.13) under conditions (2.2).

In the case of a homogeneous plane, $A=0$ and system (2.13) is divided into two independent integral differential equations. If the inclusion expands (i.e., $p_{0}(x)=0$ ), then the problem is reduced to the Prandtl-type integral differential equation

$$
\frac{\varphi(x)}{E_{1}(x)}-\frac{B}{\pi} \int_{-b}^{b} \frac{\varphi^{\prime}(t) d t}{t-x}=\frac{f_{1}(x)}{E_{1}(x)}, \quad-b<x<b,
$$

provided that $\varphi(-b)=0, \varphi(b)=T_{0}$.

The uniqueness theorem for the problem stated above can be proved analogously to the proof of Theorem 1.1.

\section{$\S 3$. Solution of a Prandtl-type Integral Differential EQUATION}

Let us consider the integral differential equation

$$
\mu(x)-\frac{\lambda}{\pi} E(x) \int_{-b}^{b} \frac{\mu^{\prime}(t) d t}{t-x}=f(x), \quad|x|<b,
$$

where $\lambda>0, E(x) \neq 0$ (except maybe $x= \pm b$ ), $f(x)$ are given functions satisfying Hölder's condition on the segment $[-b, b]$, and $\mu(x)$ is an unknown Hölder continuous function.

If the function $E(x) \equiv$ const or $E(x) \neq 0$ for all $-b \leq x \leq b$, then equation (3.1) is equivalent to a Fredholm integral equation of second kind. Assuming $\sqrt{b^{2}-x^{2}} / E(x)$ to be the entire analytic function of $x$, with the help of the solution of Riemann's problem we construct a regular integral 
equation equivalent to equation (3.1) and admitting an effective solution when $\sqrt{b^{2}-x^{2}} / E(x)$ is a polynomial [8]. In a more general case when the last function has first-order derivative satisfying Hölder's condition (except perhaps the values $x= \pm b$ ), we constructed the above-mentioned regular integral equation [9]. The obtained integral equation admits an effective solution if and only if the function $\sqrt{b^{2}-x^{2}} / E(x)$ is either a polynomial or a rational function.

In all the above-mentioned cases the function $\mu^{\prime}(x)$ possesses a square root order singularity at the points $x= \pm b$. When the function $E(x)$ has higher-order zeros $n(n>1)$ at the points $x= \pm b$, due to physical considerations, the function $\mu^{\prime}(x)$ is expected to be bounded or vanishing at the ends of the segment $[-b, b]$. Such a study is carried out in [10]. Passage in both parts of equation (3.1) to the limit respectively at the points $x= \pm b$ results in

$$
\mu( \pm b)=f( \pm b) .
$$

Conditions (3.2) are natural ones, since in the contact problems of elasticity described in Sections 1 and 2 they express the conditions of equilibrium of the inclusion.

We rewrite equation (3.1) as follows:

$$
\frac{\mu(x)-f(x)}{E(x)}-\frac{\lambda}{\pi} \int_{-b}^{b} \frac{\mu^{\prime}(t)-f^{\prime}(t)}{t-x} d t=\frac{\lambda}{\pi} \int_{-b}^{b} \frac{f^{\prime}(t) d t}{t-x}, \quad|x|<b .
$$

Introducing the notation $\mu(x)-f(x)=\nu(x)$, we obtain

$$
\frac{\nu(x)}{E(x)}-\frac{\lambda}{\pi} \int_{-b}^{b} \frac{\nu^{\prime}(t) d t}{t-x}=g(x), \quad|x|<b,
$$

where

$$
g(x)=\frac{\lambda}{\pi} \int_{-b}^{b} \frac{f^{\prime}(t) d t}{t-x} .
$$

By (3.2) the unknown function $\nu(x)$ satisfies the following supplementary conditions:

$$
\nu( \pm b)=0 .
$$

Consider now the Cauchy-type integral

$$
\Phi(x)=\frac{1}{2 \pi i} \int_{-b}^{b} \frac{\nu(t) d t}{t-x},
$$

which obviously represents a function holomorphic everywhere on the plane, except maybe the segment $[-b, b]$. 
From (3.6), taking into account conditions (3.5) and differentiating by parts, we obtain

$$
\Phi^{\prime}(x)=\frac{1}{2 \pi i} \int_{-b}^{b} \frac{\nu^{\prime}(t) d t}{t-x},
$$

On the basis of the well-known properties of Cauchy-type integrals [7], from (3.6) and (3.7) by passing to the limit we get

$$
\begin{gathered}
\nu(x)=\Phi_{+}(x)-\Phi_{-}(x), \\
\int_{-b}^{b} \frac{\nu^{\prime}(t) d t}{t-x}=\pi i\left[\Phi_{+}^{\prime}(x)+\Phi_{-}^{\prime}(x)\right],
\end{gathered}
$$

where $\Phi_{+}(x)$ and $\Phi_{-}(x)$ are respectively the limiting values of the functions $\Phi(z)$ in the upper and the lower half-plane.

We restrict ourselves to considering the case with $E(x)=\sqrt{b^{2}-x^{2}}\left(b^{2}-\right.$ $\left.x^{2}\right)^{n} P_{0}(x)$, where $n \geq 1$ is a natural number and $p_{0}(x)$ is a polynomial satisfying the condition $P_{0}(x)>0, P_{0}(-x)=P_{0}(x)$, for $-b \leq x \leq b$.

Bearing in mind the branch of $E(z)$ satisfying the condition $E_{+}(x)=$ $-E_{-}(x) \equiv E(x)>0$ for $(-b<x<b)$, by virtue of (3.8) we rewrite equation (3.3) as

$$
\Phi_{+}^{\prime}(x)+\frac{i}{\lambda E_{+}(x)} \Phi_{+}(x)+\Phi_{-}^{\prime}(x)+\frac{i}{\lambda E_{-}(x)} \Phi_{-}(x)=\frac{i g(x)}{\lambda} .
$$

Let us introduce a new function

$$
F(z)=\left[\Phi^{\prime}(z)+\frac{i}{\lambda E(z)} \Phi(z)\right] \sqrt{b^{2}-z^{2}}
$$

which is evidently holomorphic everywhere on the plane, except maybe the segment $[-b, b]$, vanishing at infinity, continuously extendable up to the internal points of the segment both from the upper and from the lower half-planes.

Then equation (3.9) takes the form

$$
F_{+}(z)-F_{-}(x)=\frac{i g(x)}{\lambda} \sqrt{b^{2}-z^{2}} .
$$

The solution of this problem can be represented as follows:

$$
F(z)=\frac{1}{2 \pi i} \int_{-b}^{b} \frac{F_{+}(t)-F_{-}(t)}{t-z} d t
$$

where the point $z$ belongs to the domain occupied by the whole plane, except maybe the segment $[-b, b]$. 
From (3.10) we have

$\Phi_{+}^{\prime}(x)+\frac{i}{\lambda E(x)} \Phi_{+}(x)=\frac{F_{+}(x)}{\sqrt{b^{2}-z^{2}}}, \quad \Phi_{-}^{\prime}(x)-\frac{i}{\lambda E(x)} \Phi_{-}(x)=-\frac{F_{-}(x)}{\sqrt{b^{2}-z^{2}}}$.

Integration of these first kind differential equations results in

$$
\begin{gathered}
\Phi_{+}(x)=\Phi_{+}(0) e^{-i \theta(x)}+\int_{0}^{x} e^{i[\theta(t)-\theta(x)]} \frac{F_{+}(t)}{\sqrt{b^{2}-z^{2}}} d t, \\
\Phi_{-}(x)=-\Phi_{-}(0) e^{i \theta(x)}-\int_{0}^{x} e^{-i[\theta(t)-\theta(x)]} \frac{F_{-}(t)}{\sqrt{b^{2}-z^{2}}} d t
\end{gathered}
$$

where $\theta=\frac{1}{\lambda} \int_{0}^{x} \frac{d t}{E(t)},|x|<b$.

According to (3.8) and (3.13) we have

$$
\begin{aligned}
& \nu(x)=\nu(0) \cos \theta(x)+\nu_{1} \sin \theta(x)+\int_{0}^{x} \frac{\cos (\theta(t)-\theta(x))}{\sqrt{b^{2}-t^{2}}}\left[F_{+}(t)+F_{-}(t)\right] d t+ \\
& +i \int_{0}^{x} \frac{\sin (\theta(t)-\theta(x))}{\sqrt{b^{2}-t^{2}}}\left[F_{+}(t)-F_{-}(t)\right] d t, \text { where } \nu_{1}=-\frac{1}{\pi} \int_{-b}^{b} \frac{\nu(t) d t}{t} .
\end{aligned}
$$

Owing to (3.8), the last expression takes the form

$$
\begin{aligned}
\nu(x) & =\nu(0) \cos \theta(x)+\nu_{1} \sin \theta(x)+\int_{0}^{x} \frac{A(t) \sin (\theta(t)-\theta(x))}{\sqrt{b^{2}-t^{2}}} d t+ \\
& +\int_{0}^{x} \frac{B(t) \cos (\theta(t)-\theta(x))}{\sqrt{b^{2}-t^{2}}} d t
\end{aligned}
$$

where

$$
A(x)=-\frac{g(x)}{\lambda} \sqrt{b^{2}-x^{2}}, \quad B(x)=\frac{1}{\pi \lambda} \int_{-b}^{b} \frac{g(t) \sqrt{b^{2}-t^{2}}}{t-x} d t
$$

Representing formula (3.14) in terms of

$$
\begin{aligned}
\nu(x) & =\left[\nu(0)+\int_{0}^{x} \frac{A(t)}{\sqrt{b^{2}-t^{2}}} \sin \theta(t) d t+\int_{0}^{x} \frac{B(t)}{\sqrt{b^{2}-t^{2}}} \cos \theta(t) d t\right] \cos \theta(x)+ \\
+ & {\left[\nu_{1}-\int_{0}^{x} \frac{A(t)}{\sqrt{b^{2}-t^{2}}} \cos \theta(t) d t+\int_{0}^{x} \frac{B(t)}{\sqrt{b^{2}-t^{2}}} \sin \theta(t) d t\right] \sin \theta(x), }
\end{aligned}
$$

and taking into consideration that the functions $\sin \theta(x)$ and $\cos \theta(x)$ have no limit at the points $x= \pm b$, we conclude that for condition (3.5) to 
be fulfilled it is necessary and sufficient that the following conditions be fulfilled:

$$
\begin{aligned}
& \lim _{x \rightarrow \pm b}\left[\nu(0)+\int_{0}^{x} \frac{A(t) \sin \theta(t)}{\sqrt{b^{2}-t^{2}}} d t+\int_{0}^{x} \frac{B(t) \cos \theta(x)}{\sqrt{b^{2}-t^{2}}} d t\right]=0, \\
& \lim _{x \rightarrow \pm b}\left[\nu_{1}-\int_{0}^{x} \frac{A(t) \cos \theta(t)}{\sqrt{b^{2}-t^{2}}} d t+\int_{0}^{x} \frac{B(t) \sin \theta(x)}{\sqrt{b^{2}-t^{2}}} d t\right]=0 .
\end{aligned}
$$

Thus we have proved

Lemma.For the solution of equation (3.1) to satisfy conditions (3.5) it is necessary and sufficient that the function $f(x)$ satisfy conditions (3.17).

By virtue of (3.4), (3.15) we can conclude that for conditions (3.17) to be fulfilled it is sufficient that the function $f(x)$ be even (odd), whence it follows that the unknown function $\mu(x)$ is even (odd).

In particular, if the function $f(x)$ is even, then from (3.17) we obtain

$$
\nu(0)=-\int_{0}^{b} \frac{A(t) \sin \theta(t)}{\sqrt{b^{2}-t^{2}}} d t+\int_{0}^{b} \frac{B(t) \cos \theta(x)}{\sqrt{b^{2}-t^{2}}}, \quad \nu_{1}=0,
$$

but if the function $f(x)$ is odd, then

$$
\nu(0)=0, \quad \nu_{1}=\int_{0}^{b} \frac{A(t) \cos \theta(t)}{\sqrt{b^{2}-t^{2}}} d t-\int_{0}^{b} \frac{B(t) \sin \theta(x)}{\sqrt{b^{2}-t^{2}}} .
$$

To determine the asymptotic behavior of the function $\nu^{\prime}(x)$ let us perform some simple transformations in formula (3.16):

$$
\begin{aligned}
\nu(x) & =\left[\nu(0)+\lambda A_{0}(0)-\lambda^{2} B_{1}(0)-\lambda^{2} \int_{0}^{x} A_{1}^{\prime}(t) \sin \theta(t) d t-\right. \\
& \left.-\lambda^{2} \int_{0}^{x} B_{1}^{\prime}(t) \cos \theta(t) d t\right] \cos \theta(x)+\left[\nu_{1}+\lambda B_{0}(0)+\lambda^{2} A_{1}(0)+\right. \\
& \left.+\lambda^{2} \int_{0}^{x} A_{1}^{\prime}(t) \cos \theta(t) d t-\lambda^{2} \int_{0}^{x} B_{1}^{\prime}(t) \sin \theta(t) d t\right] \sin \theta(x)- \\
& -\lambda A_{0}(x)+\lambda^{2} B_{1}(x)
\end{aligned}
$$

where

$$
\begin{array}{ll}
A_{0}(x)=\frac{A(x) E(x)}{\sqrt{b^{2}-x^{2}}}, & A_{1}(x)=A_{0}^{\prime}(x) E(x), \\
B_{0}(x)=\frac{B(x) E(x)}{\sqrt{b^{2}-x^{2}}}, & B_{1}(x)=B_{0}^{\prime}(x) E(x) .
\end{array}
$$

The function $\nu^{\prime}(x)$ has the form

$$
\nu^{\prime}(x)=\left[-\lambda^{2} A_{1}^{\prime}(x) \sin \theta(x)-\lambda^{2} B_{1}^{\prime}(x) \cos \theta(x)\right] \cos \theta(x)+
$$




$$
\begin{aligned}
& +\left[\lambda^{2} A_{1}^{\prime}(x) \cos \theta(x)-\lambda^{2} B_{1}^{\prime}(t) \sin \theta(x)\right] \sin \theta(x)- \\
& -\frac{P(x)}{\lambda E(x)} \sin \theta(x)+\frac{R(x)}{\lambda E(x)} \cos \theta(x)-\lambda A_{0}^{\prime}(x)+\lambda^{2} B_{1}^{\prime}(x)= \\
& =-\lambda A_{0}^{\prime}(x)-\frac{P(x)}{\lambda E(x)} \sin \theta(x)+\frac{R(x)}{\lambda E(x)} \cos \theta(x),
\end{aligned}
$$

where

$$
\begin{aligned}
P(x) & =\nu(0)+\lambda A_{0}(0)-\lambda^{2} B_{1}(0)-\lambda^{2} \int_{0}^{x} A_{1}^{\prime}(t) \sin \theta(t) d t- \\
& -\lambda^{2} \int_{0}^{x} B_{1}^{\prime}(t) \cos \theta(t) d t \\
R(x) & =\nu_{1}+\lambda B_{0}(0)+\lambda^{2} A_{1}(0)+\lambda^{2} \int_{0}^{x} A_{1}^{\prime}(t) \cos \theta(t) d t- \\
& -\lambda^{2} \int_{0}^{x} B_{1}^{\prime}(t) \sin \theta(t) d t .
\end{aligned}
$$

Taking into account conditions (3.17) and also formulas (3.18), we obtain

$$
\lim _{x \rightarrow \pm b} P(x)=0, \quad \lim _{x \rightarrow \pm b} R(x)=0 .
$$

Let $f^{\prime}(x)$ be a Hölder-continuous function on the segment $[-b, b]$ and let $f^{\prime}( \pm b)=0$. Then by virtue of formulas (3.3), (3.15), and (3.18) we arrive at the following expressions: $A_{0}^{\prime}(x)=O\left(\left(b^{2}-x^{2}\right)\right)^{n-\frac{1}{2}}, B_{0}^{\prime}(x)=O\left(\left(b^{2}-x^{2}\right)\right)^{n}$ in the vicinity of the points $x= \pm b$. If $f^{\prime}( \pm b) \neq 0$, then we have $A_{0}^{\prime}(x)=$ $O\left(\left(b^{2}-x^{2}\right)^{n-\frac{1}{2}} \ln \frac{b-x}{b+x}\right), B_{0}^{\prime}(x)=O\left(\left(b^{2}-x^{2}\right)^{n} \ln \frac{b-x}{b+x}\right)$ in the vicinity of the points $x= \pm b$.

If $f^{\prime}(x) \in H^{*}(-b, b)$, i.e., it has a singularity of integrable order at the points $x= \pm b: f^{\prime}(x)=\frac{f_{0}(x)}{\left(b^{2}-x^{2}\right)^{\alpha}}$, where $0<\alpha<1, f_{0}(x)$ belongs to the Hölder class in a vicinity of the points $x= \pm b$, then by an analogous reasoning we get $A_{0}^{\prime}(x)=O\left(\left(b^{2}-x^{2}\right)^{n-\alpha-\frac{1}{2}}\right), B_{0}^{\prime}(x)=O\left(\left(b^{2}-x^{2}\right)^{n-\alpha}\right)$.

Taking into consideration (3.20) and applying l'Hospital's rule, useful in evaluating indeterminate forms, we have

$$
\begin{gathered}
\lim _{x \rightarrow \pm b} \frac{P(x)}{E(x)}=\lim _{x \rightarrow \pm b} \frac{-\lambda^{2} A_{1}^{\prime}(x) \sin \theta(x)-\lambda^{2} B_{1}^{\prime}(x) \cos \theta(x)}{E^{\prime}(x)}=0, \\
\text { i.e. } \frac{P(x)}{E(x)}=\left(b^{2}-x^{2}\right)^{n-\beta} \ln ^{m}\left(\frac{b-x}{b+x}\right) \alpha(x), \\
\lim _{x \rightarrow \pm b} \frac{R(x)}{E(x)}=\lim _{x \rightarrow \pm b} \frac{\lambda^{2} A_{1}^{\prime}(x) \cos \theta(x)-\lambda^{2} B_{1}^{\prime}(x) \sin \theta(x)}{E^{\prime}(x)}=0, \\
\text { i.e. } \frac{R(x)}{E(x)}=\left(b^{2}-x^{2}\right)^{n-\beta} \ln ^{m}\left(\frac{b-x}{b+x}\right) \beta(x),
\end{gathered}
$$


where $\alpha(x)$ and $\beta(x)$ are bounded functions in the vicinity of the points $x= \pm b, \alpha(x) \neq 0, \beta(x) \neq 0$ for $x= \pm b, \frac{1}{2} \leq \beta<\frac{3}{2} ; m=0$ for $f^{\prime}( \pm b)=0$ or $f^{\prime}(x) \in H^{*}(-b, b), m=1$ for $f^{\prime}( \pm b) \neq 0$.

Thus the following theorems are proved.

Theorem 3.1. If the function $f^{\prime}(x)$ belongs to the Hölder class on the segment $[-b, b]$ and $f^{\prime}( \pm b)=0$, then equation (3.3) has a solution which can be represented by formula (3.14), and in the vicinity of the points $x= \pm b$ the representation $\nu^{\prime}(x)=O\left(\left(b^{2}-x^{2}\right)^{n-\frac{1}{2}}\right)$ is valid.

Theorem 3.2. If the function $f^{\prime}(x)$ belongs to the Hölder class on the segment $[-b, b]$ and $f^{\prime}( \pm b) \neq 0$, then equation (3.3) has a solution which can be represented by formula (3.14), and in the vicinity of the points $x= \pm b$ we have the representation

$$
\nu^{\prime}(x)=O\left(\left(b^{2}-x^{2}\right)^{n-\frac{1}{2}} \ln \left(\frac{b-x}{b+x}\right)\right) .
$$

Theorem 3.3. If the function $f^{\prime}(x)$ belongs to the Hölder class in the interval $(-b, b)$ and at the points $x= \pm b$ it has a singularity of order $\alpha$ with $0<\alpha<1$, then equation (3.3) has a solution which can be represented by formula (3.14), and in the vicinity of the points $x= \pm b$ the estimate

$$
\nu^{\prime}(x)=O\left(\left(b^{2}-x^{2}\right)^{n-\beta}\right) \text { with } 1 / 2<\beta<3 / 2
$$

is valid.

Thus for the function $\mu^{\prime}(x)$ the following theorem holds.

Theorem 3.4. If the right-hand side of the integral differential equation (3.1) satisfies the condition: if $f^{\prime}(x)$ belongs to the Hölder class on the segment $[-b, b]$ or $f^{\prime}(x) \in H^{*}(-b, b)$, then equation (3.1) has a solution in the vicinity of the points $x= \pm b$ which can be represented as

$$
\mu^{\prime}(x)=f^{\prime}(x)+\left(b^{2}-x^{2}\right)^{n-\beta} \ln ^{m}\left(\frac{b-x}{b+x}\right) \gamma(x),
$$

where $\frac{1}{2} \leq \beta<\frac{3}{2}, \gamma(x)$ is the bounded function in the vicinity of the points $x= \pm b, \gamma(x) \neq 0$ for $x= \pm b, m=0$ or $m=1$.

Consequently, in problems of contact interaction of the theory of elasticity described in Sections 1 and 2 we have stated that the jump of tangential contact stress between the upper and the lower end of the inclusion can be bounded or can tend to zero in the vicinity of the inclusion ends.

\section{References}


1. Hwu Chyanbin and T. C. T. Ting, Two-dimensional problems of an anisotropic elastic solid with an elliptic inclusion. Quart. J. Mech. Appl. Math. 42(1989), No. 4, 553-572.

2. T. C. T. Ting and Yan Gongpu, The anisotropic elastic solid with an elliptic hole or rigid inclusion. Int. J. Solids and Str. 27(1991), No. 15, 1879-1891.

3. Y. Z. Chen, Investigation of the stress singularity coefficient for a finite plate containing a rigid line. Eng. Fract. Mech. 40(1991), No. 1, 17-24.

4. G. H. Maikap and P. R. Sengupta, Two-dimensional inclusions in an anisotropic elastic solid. Sci. Phys. Sci. 2(1990), No. 1, 31-45.

5. V. M. Alexandrov and S. M. Mkhitaryan, Contact problems for bodies with thin coverings and layers. (Russian) Nauka, Moscow, 1983.

6. G. Ja. Popov, Concentration of elastic stresses near punches, cuts, inclusions, and supports. (Russian) Nauka, Moscow, 1982.

7. N. I. Muskhelishvili, Some basic problems of the mathematical theory of elasticity. Translated from the Russian, P. Noordhoff, Groningen, 1953.

8. I. N. Vekua, On Prandtl's integral differential equation. (Russian) Prikl. Mat. Mekh. 9(1945), No. 2, 143-150.

9. L. G. Magnaradze, On one new integral equation of the theory of an aircraft wing. (Russian) Bull. Acad. Sci. Georgian SSR 3(1980), No. 6, 503-508.

10. N. N. Shavlakadze, Investigation of one type of integral differential equation and contact problems of the theory of elasticity associated with it. (Russian) Trudy Tbilis. Mat. Inst. Razmadze 105(1995), 98-107.

(Received 18.03.1996)

Author's address:

A. Razmadze Mathematical Institute

Georgian Academy of Sciences

1, M. Aleksidze St., Tbilisi 380093

Georgia 